\title{
Reproductive Aspects of the Mosquito Culex quinquefasciatus (Diptera:Culicidae) Infected with Wuchereria bancrofti (Spirurida: Onchocercidae)
}

\author{
Catarina A Lima, Walkiria R Almeida, Hilary Hurd*, Cleide MR Albuquerque/ ${ }^{+}$ \\ Departamento de Zoologia, Universidade Federal de Pernambuco, Av. Moraes Rego s/noํ, 50670-420 Recife PE, Brasil \\ *Centre for Applied Entomology and Parasitology, School of Life Sciences, Keele University, Staffordshire, UK
}

This study reports on the relationship between Wuchereria bancrofti infection and female body size, intake of blood and fecundity in the mosquito Culex quinquefasciatus, vector of this filarial parasite in Recife (Brazil). Adults from field collected larvae were infected via a membrane feeding procedure, using blood with parasitaemia ranging from 724-6,000 mf/ml. A positive correlation was observed between mosquito size (measured by wing length) and egg production in uninfected females. However, this relationship did not exist in $\mathrm{W}$. bancrofti infected mosquitoes. This change is unlikely to be the result of changes in blood ingestion as no significant difference was found when infected and uninfected females were compared. Variation in egg production observed between trials could not be associated with parasite density in the blood. These results suggest infection with $\mathrm{W}$. bancrofti may disrupt the relationship between mosquito size and egg production during the first gonotrophic cycle of C. quinquefasciatus such that fecundity is sometimes reduced. However, this overall affect is variable and many groups of mosquitoes do not respond in this way.

Key words: Wuchereria bancrofti - Culex quinquefasciatus - mosquito - fecundity - body size - blood feeding

The parasite Wuchereria bancrofti is distributed throughout tropical regions (Central and South America, Africa, Asia, Pacific areas) and also in subtropical countries in the Middle East (Service 1996). Current estimates (Ottesen \& Ramachandran 1995) suggest that approximately 120 million people are infected with all types of lymphatic filariae, particularly bancroftian filariasis. In Recife, a Brazilian coastal city (on 8०4'3”' south latitude) bancroftian filariasis is endemic in many areas and is transmitted by Culex quinquefasciatus. The high fecundity of this mosquito species, together with the increase in number of breeding places due to uncontrolled urbanization, may have contributed to the recent increase in prevalence rates of W. bancrofti from 9.7\% (Azevedo \& Dobbin 1952) up to $14.9 \%$ (Maciel et al. 1996).

Previous work in our laboratory has shown that $C$. quinquefasciatus (Recife strain) is able to control the number of developing $W$. bancrofti (1-2/mosquito) when ingesting variable densities of parasite up to $2,600 \mathrm{mi}-$ crofilariae (mf) per ml (Albuquerque et al. 1999). These observations suggest that there is a regulation mechanism that controls the number of infective larvae that can successfully mature in C. quinquefasciatus. This mechanism is likely to involve a defence response although en-

Financial support: Wellcome Trust via a Research Development Award in Tropical Medicine (047085 to CRMA) and CNPq (process no. 521126/98-3).

${ }^{+}$Corresponding author. Fax: +55-81-271.8359. E-mail: cleide@ufpe.br

Received 18 June 2002

Accepted 14 November 2002 capsulated and/or melanized $\mathrm{mf}$ are rarely observed. Whatever the method of parasite elimination, we expect it to be costly to the mosquito in terms of resources (Sheldon \& Verhulst 1996), as will repair to the damage to the midgut caused by mf invasion.

Observations from several parasite/insect systems have shown that infection costs can manifest as a reduction in host reproductive fitness or longevity and that fecundity reduction may occur very early in the infection, before the developing parasites are likely to cause a drain on host metabolic resources (reviewed by Hurd \& Webb 1997).

Of particular relevance to this study are examples of reduction in the reproductive output of mosquitoes resulting from protozoan (for example, Hogg \& Hurd 1995a, b, 1997) and nematode infections (Javadian \& Macdonald 1974, Christensen 1981, Ferdig et al. 1993). In Anopheles gambiae, a reduction of $41.2 \%$ in fecundity and $61.8 \%$ in fertility was observed in mosquitoes infected with rodent malaria parasite Plasmodium yoelii nigeriensis (Ahmed et al. 1999). Parasite-induced fecundity reduction in Aedes trivittatus was related to the number of Dirofilaria immitis it harboured, with a severe reduction in egg production occurring in females infected with more than 15 filarial parasites (Christensen 1981).

We wished to determine whether $W$. bancrofti has a similar effect on the fecundity and fertility of $C$. quinquefasciatus during the first gonotrophic cycle postinfection, when $\mathrm{mf}$ are penetrating the midgut, traversing the haemocoel and invading the thoracic muscles. It is during this 4-7 day period that the mosquito is able to eliminate the majority of invading $\mathrm{mf}$ by a mechanism as yet undescribed (Albuquerque et al. 1999). The aim in this work was, therefore, to assess the impact of exposure to different levels of $W$. bancrofti $\mathrm{mf}$ on the reproductive 
fitness of $C$. quinquefasciatus. In addition, factors known to affect the fecundity, such as body size (Colless \& Chellapah 1960, Briegel 1990) and bloodmeal size (Lea et al. 1978), were investigated to assess the possibility that they might contribute to any fecundity reduction observed in infected females.

\section{MATERIALS AND METHODS}

Mosquito collection - C. quinquefasciatus larvae were collected from the filariasis endemic area of Azeitona, located in the district of the Metropolitan Zone in Recife, PE, Brazil. Collections were made in the same habitat, a moderately shaded drainage ditch $40 \mathrm{~m}$ long and $0.5 \mathrm{~m}$ wide. Samples were collected and transported to the laboratory where last stage larvae (L4) were separated from the associated fauna and were preliminary screened for species identification based on external morphological characters (Forattini 1996). Male and female mosquitoes emerging within a $24 \mathrm{~h}$ period were kept together for mating and were provided with sugar lumps and cotton pads soaked in water. Mosquito adults were maintained at $27 \pm$ $3^{\circ} \mathrm{C}, 85 \pm 10 \%$ humidity in a $12: 12 \mathrm{~h}$ light/dark cycle. Before adult dissection, species confirmation was carried out based on diagnose from Consoli and Oliveira (1994).

Infection via artificial blood feeding - Artificial feeding was carried out as previously described by Albuquerque et al. (1999) using three to four day old female mosquitoes. Blood with different densities of circulating parasites, ranging between 724-6000 mf/ml, was used in this work. Blood was obtained from human lymphatic filariasis carriers, who participated in a survey carried out by Centro de Pesquisa Aggeu Magalhães-Fiocruz, Recife, after written informed consent. Venous blood was collected between 23.00 and $24.00 \mathrm{~h}$ in vacutainers containing EDTA $(1 \mathrm{mg} / \mathrm{ml})$ as an anticoagulant. At the end of the work, donors were treated with diethylcarbamazine according to the therapeutic recommendation of the World Health Organization (WHO 1987). Microfilarial density was estimated by counting the number in $60 \mathrm{~mm}^{3}\left(3 \times 20 \mathrm{~mm}^{3}\right.$ aliquots) of blood and expressed as $\mathrm{mf} / \mathrm{ml}$. Groups of mosquitoes were allowed to feed for $30 \mathrm{~min}$ on the infected blood, heated to $37^{\circ} \mathrm{C}$, using glass feeders covered with cow gut membrane. To ensure mf homogeneity the blood was kept agitated using a stirrer at $1 \mathrm{red} / \mathrm{sec}$. After feeding, individual females were transferred to universal tubes and maintained for 3 days for the total excretion of haematin, used to estimate the blood meal size. Only females that were fully engorged were used in the experiments. A control group of mosquitoes was fed on uninfected human blood.

All mosquitoes were dissected to check for the presence of $\mathrm{mf}$ in the thoracic muscles at the time of ovary dissection (4-7 days post blood meal). A subsample of infected mosquitoes from all groups was dissected immediately post infection to confirm that microfilariae had been ingested.

Haematin assay - Assessment of mosquito bloodmeal size by haemoglobinometry (Briegel et al. 1979) was not feasible, as this technique requires killing the insect before egg production occurs. Haemoglobin intake was thus measured indirectly by making a spectrophotometric de- termination of the amount of haematin excreted by each mosquito. Haematin analysis was performed by dissolving the excretory products, deposited in the tubes, in $1 \mathrm{ml}$ of $1 \%$ lithium carbonate. The absorbance was measured at $395 \mathrm{~nm}$ and the amount of haematin present determined by comparison with bovine haematin standards (Briegel 1980, Hogg \& Hurd 1997).

Assessment of egg production and fertility - Following blood meal digestion mosquitoes were moved from the first vial to oviposition tubes contain $1.5 \mathrm{ml}$ of water previously used to culture larvae. The number of eggs laid by each surviving female was recorded from 4 to 7 days post blood meal. Retention of fully developed eggs was determined following ovary dissection after oviposition, to observe the presence of any retained eggs. Total egg production was considered as the sum of laid and retained eggs from the first gonotrophic cycle. Fertility was measured as number of larvae hatched from each raft.

Mosquito size - Mosquito body-size was expressed in terms of wing length. One wing was selected from each mosquito, mounted on a slide, and its length measured from the tip, excluding fringe, to the distal end of the allula (Lyimo \& Koella 1992) using a calibrated dissecting microscope with an ocular micrometer.

Statistical analysis - Data collected on wing length, haematin excretion and egg production were tested for normality. A comparison of egg production, blood meal size and wing length between different parasitaemia levels and between infected and uninfected groups within the same parasitaemia level was made by multiple analysis of variance (MANOVA) and the LSD post hoc test. A $p$ value of $<0.05 \%$ was accepted as significant. Comparisons of percentage survivorship and fertility between uninfected and infected groups were assessed by Chisquare analysis. The correlation of blood meal size and wing length on egg production within species was determined by regression analysis. All statistical tests were performed using Statistic 5.0 and Bio Estatistic 6.0 versions. All mean values were expressed \pm standard deviations.

\section{RESULTS}

The effect of filarial infection on mosquito fecundity was assessed in eight experiments using mf density ranging from 724 to $6,000 \mathrm{mf} / \mathrm{ml}$ (Table I). Two of these experiments (blood samples with mf densities of 800 and 6,000 respectively) were performed on groups of mosquitoes from the same generation and of the same age in order to compare the effect of different parasite densities on the fecundity of mosquitoes that developed in the same situation. From a total of 421 females that laid eggs following an infective blood meal $48.9 \%(n=207)$ were found to be infected and were used in our assays. As with our previous investigation (Albuquerque et al. 1999) a small subset of mosquitoes from each trial (group infected with $6,000 \mathrm{mf} / \mathrm{ml}$ not assessed) showed that the average number of larvae recovered 4-7 days post an infective blood meal (period of observation) varied from 1-2 per infected female. In each experiment, mosquitoes ingesting a control blood meal, fed on blood taken from the same uninfected subject $(n=350)$. 
TABLE I

Eggs production by Culex quinquefasciatus uninfected and infected with Wuchereria bancrofti at different microfilariae (mf) rate in the blood

\begin{tabular}{lllcc}
\hline \multirow{2}{*}{$\begin{array}{l}\text { Mf density in } \\
\text { the blood/ml }\end{array}$} & \multicolumn{4}{c}{ Mean no. of eggs produced/female \pm SD } \\
\cline { 2 - 6 } & Infected & $\mathrm{N}$ & Uninfected & $\mathrm{N}$ \\
\hline 724 & $96.40 \pm 20.90^{a}$ & $(25)$ & $79.50 \pm 27.60$ & $(28)$ \\
$800^{c}$ & $72.31 \pm 18.61$ & $(19)$ & $79.95 \pm 18.49$ & $(49)$ \\
1150 & $97.20 \pm 22.90^{a}$ & $(27)$ & $86.80 \pm 18.40$ & $(47)$ \\
1200 & $66.50 \pm 14.70$ & $(20)$ & $66.60 \pm 17.90$ & $(46)$ \\
1500 & $72.40 \pm 16.50^{b}$ & $(30)$ & $85.50 \pm 19.60$ & $(45)$ \\
1750 & $68.07 \pm 20.60$ & $(14)$ & $69.26 \pm 24.47$ & $(15)$ \\
2900 & $82.50 \pm 17.90^{b}$ & $(32)$ & $97.10 \pm 16.40$ & $(71)$ \\
$6000^{c}$ & $72.32 \pm 17.80$ & $(40)$ & $79.95 \pm 18.40$ & $(49)$ \\
\hline Total & \multicolumn{5}{c}{$(207)$} \\
\hline
\end{tabular}

$a$ : significantly greater than the respective control $(\mathrm{P}<0.05) ; b$ : significantly less than the respective control $(\mathrm{P}<0.05) ; c$ : infected mosquitoes in these trials were from the same generation but fed on different blood sources from subjects with different parasitaemias; $\mathrm{N}$ : sample size

Mean wing size of each group was assessed in five of the trials (see Table II). Body size ranged from 3.69-4.25 $\mathrm{mm}$ and 3.72-4.22 $\mathrm{mm}$ in infected and uninfected females respectively. Some variation in size occurred throughout the collection period (one year), probably as a result of changes in environmental conditions at the collection site. However, when data from uninfected and infected mosquitoes were analyzed via pair wise comparisons, using the Scheffé test at $0.05 \%$, no significant differences were detected between the mosquitoes assigned to infected or control groups in any individual trial (Table II). Thus size differences could not account for differences in egg production. Likewise, statistically significant differences in blood meal size (as measured by the amount of haematin excreted) were not observed when infected and control females were compared in any of the individual trials (Table II). Blood meal sizes were not affected by the density of $\mathrm{mf}$ in the blood meal used for feeding in these five trials.

As expected, mosquito size showed a significant overall positive correlation with egg production by linear regression when mosquitoes fed on uninfected blood $(\mathrm{R}=$
$0.38, \mathrm{P}=0.0001)$. However, the presence of $\mathrm{mf}$ destroyed this relationship and no positive correlation between mosquito size and egg production was observed in the infected group $(\mathrm{R}=0.12, \mathrm{P}=0.06)$ (Fig. $1 \mathrm{a}, \mathrm{b})$.

Considerable variation in haematin excretion occurred in all 5 trials and although, overall, a positive relationship was observed between haematin excretion and egg production in mosquitoes fed on uninfected blood, this was not a significant correlation (at $\mathrm{p}=0.05$ level) $(\mathrm{R}=0.12 ; \mathrm{P}$ $=0.06 ; \mathrm{N}=236)$. No relationship was demonstrated for
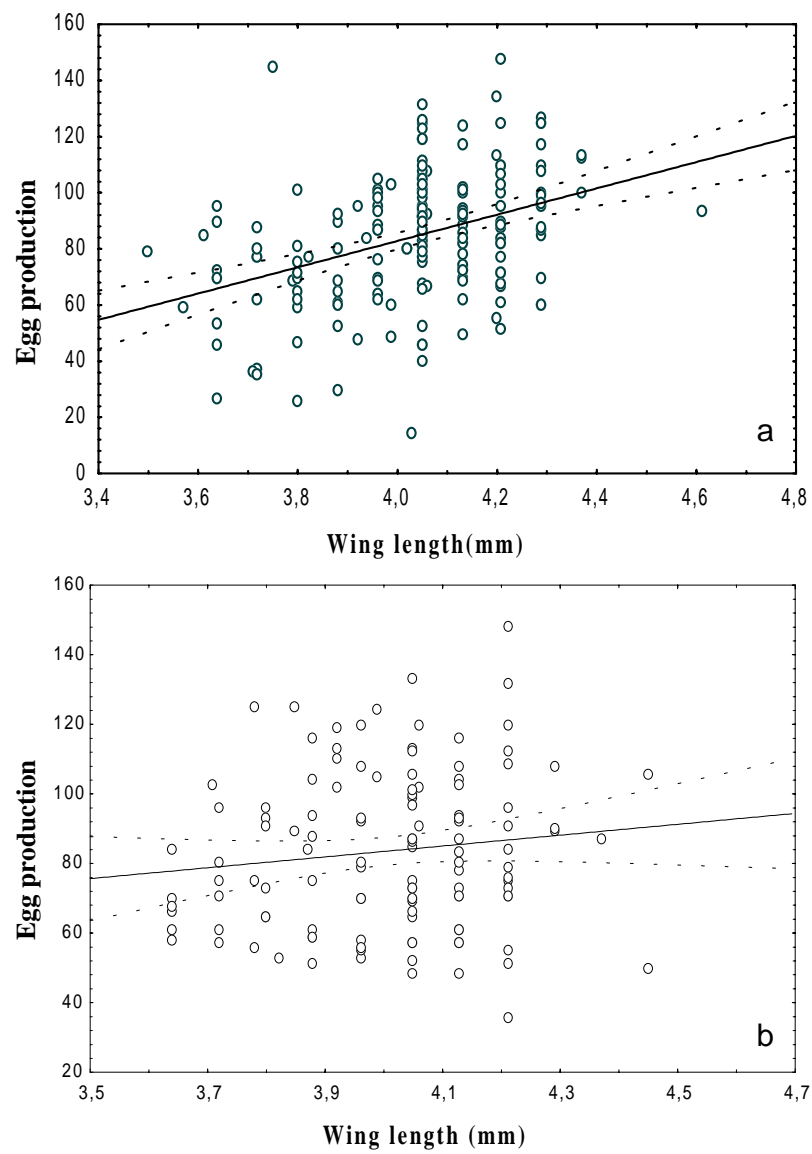

Fig. 1: linear regression of body size against egg production, for Culex quinquefasciatus. a: uninfected group $(\mathrm{y}=-104+46.83 \mathrm{x} ; \mathrm{N}$ $=205) ; \mathrm{b}$ : infected group $(\mathrm{y}=21.01+15.61 \mathrm{x} ; \mathrm{N}=123)$

TABLE II

Wing size and haematin excretion of Culex quinquefasciatus infected with Wuchereria bancrofti. Females were fed on blood with different parasitaemia levels

\begin{tabular}{|c|c|c|c|c|c|c|}
\hline \multirow[b]{2}{*}{$\mathrm{Mf} / \mathrm{ml}$} & \multicolumn{2}{|c|}{$\begin{array}{l}\text { Wing size }(\mathrm{mm}) \\
\mathrm{X} \pm \mathrm{DP}(\mathrm{N})\end{array}$} & \multirow[b]{2}{*}{$\mathrm{p}$} & \multicolumn{2}{|c|}{$\begin{array}{c}\text { Haematin }(\mu \mathrm{g}) \\
\mathrm{X} \pm \mathrm{DP}(\mathrm{N})\end{array}$} & \multirow[b]{2}{*}{$\mathrm{p}$} \\
\hline & Infected & Controls & & Infected & Controls & \\
\hline 724 & $3.89 \pm 0.12(18)$ & $3.94 \pm 0.21(21)$ & 0.99 & $15.10 \pm 2.37(25)$ & $14.70 \pm 2.80$ & 0.40 \\
\hline 1150 & $4.00 \pm 0.16(25)$ & $4.06 \pm 0.12(30)$ & 0.98 & $15.80 \pm 1.52(27)$ & $14.60 \pm 0.97(47)$ & 0.99 \\
\hline 1200 & $3.82 \pm 0.13(20)$ & $3.90 \pm 0.18$ & 0.93 & $14.70 \pm 1.94(20)$ & $15.10 \pm 0.97(46)$ & 0.99 \\
\hline 1500 & $4.07 \pm 0.15(28)$ & $4.08 \pm 0.19$ & 1.00 & $14.60 \pm 2.98(30)$ & $15.70 \pm 2.21(45)$ & 0.98 \\
\hline 2900 & $4.14 \pm 0.11(32)$ & $4.10 \pm 0.12(65)$ & 0.99 & $15.80 \pm 0.65(32)$ & $14.90 \pm 1.86(70)$ & 0.99 \\
\hline Total & 123 & 205 & & 134 & 236 & \\
\hline
\end{tabular}


mosquitoes feeding on infected blood $(\mathrm{R}=0.04 ; \mathrm{P}=0.63$; $N=134$ ) (data not shown) Similarly, although haematin production increased with mosquito size, this relationship was not significant in this study (Fig. 2 a, b).

Although analysis of the total data set from 5 trials demonstrated that infection disrupted the usual relationship between size and reproductive output, comparison of mean egg production by uninfected and infected females in all eight individual trials did not produce a consistent picture (see Table I). Comparisons between infected groups and their respective controls showed that significantly fewer eggs were produced by infected females fed on blood containing 1,500 and $2,900 \mathrm{mf} / \mathrm{ml}$. In contrast, a significant increase in the number of eggs produced occurred in infected females fed on blood with parasitaemia levels of 724 and $1,150 \mathrm{mf} / \mathrm{ml}$ and no statistically significant difference between infected and uninfected mosquitoes was observed in the other four experiments.

Similar results were observed when the number of eggs produced by infected females and those submitted to an infected blood meal but remaining uninfected were compared. At the time of dissection, it was impossible to determine whether mosquitoes in the latter group had been infected and eliminated the parasite or whether no para-
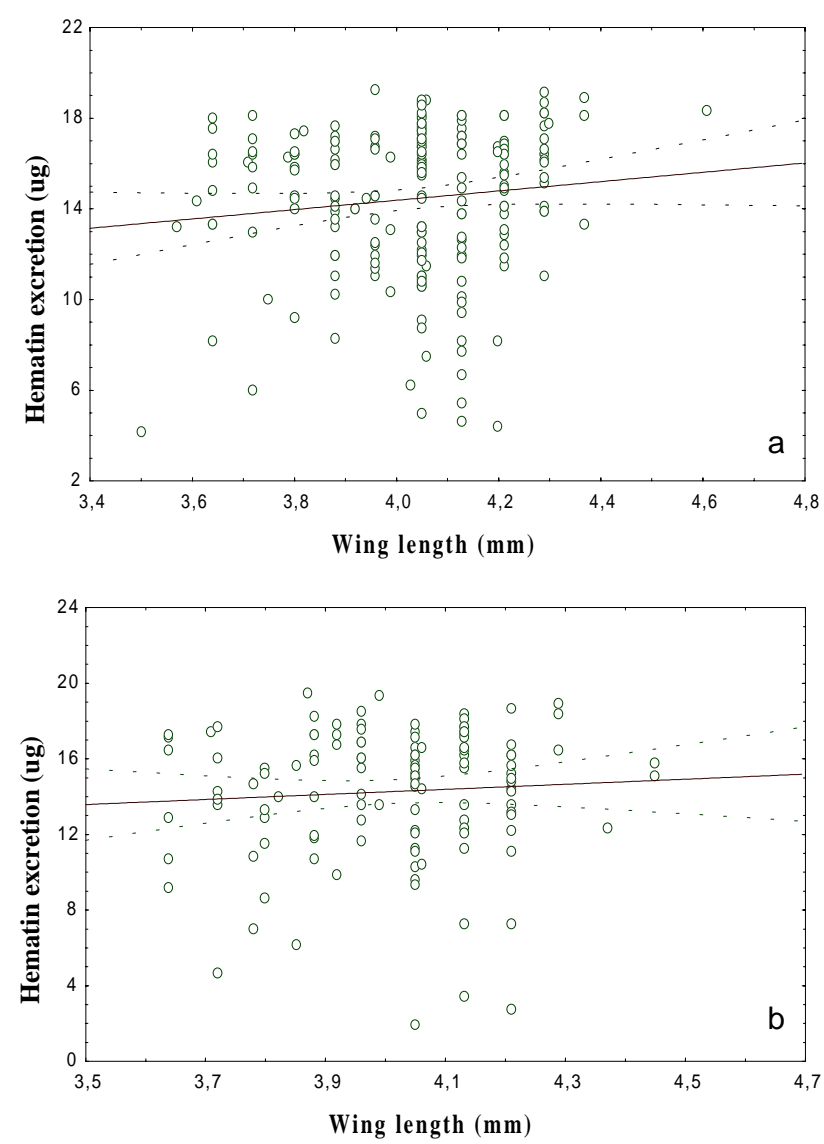

Fig. 2. linear regression of body size against hematin excretion from Culex quinquefasciatus. a: untreated group ( $\mathrm{R}=0.11, p=$ $0.093, \mathrm{~N}=205 ; \mathrm{y}=6.15+2.05 \mathrm{x}) ; \mathrm{b}$ : infected group $(\mathrm{R}=0.06, p$ $=0.45, \mathrm{~N}=123 ; \mathrm{y}=8.89+1.34 \mathrm{x})$. site had been ingested during bloodfeeding. Data from these uninfected mosquitoes were therefore removed from the study when the effect of the parasite on egg production was examined.

Our comparison of egg production by mosquitoes from the same generation, but feeding on blood with different $\mathrm{mf}$ densities $(800$ and $6,000 \mathrm{mf} / \mathrm{ml})$ revealed that a similar number of eggs was produced by infected females in each group (Table I), confirming that parasite density in the blood meal did not affect egg production

The presence of the parasite in the blood had no effect on egg retention in the oviduct, which was minimal and only observed at parasitaemia of $724 \mathrm{mf} / \mathrm{ml}(0.4 \%$ of eggs) and $1,500 \mathrm{mf} / \mathrm{ml}(0.5 \%$ of eggs). In mosquitoes fed on uninfected blood, $0.4 \%$ of eggs produced were not laid within the 7 days post blood meal.

Data for fertility was only obtained for the experiment with $2,900 \mathrm{mf} / \mathrm{ml}$. Although total egg production was significantly reduced by infection in this trial, ingestion of an infected blood meal did not significantly reduce egg fertility, as measured by the hatching rate of eggs laid by infected $(94.2 \%, \mathrm{~N}=1,799 \mathrm{eggs})$ and uninfected females $(93 \%, \mathrm{~N}=1,373 \mathrm{eggs})$, respectively $\left(\chi^{2}=0.064, \mathrm{df}=1, \mathrm{P}=\right.$ $0.823)$.

The prevalence of infection was strongly affected by mosquito size (Fig. 3). The greatest proportion of mosquitoes with one or more worms fell within the mid size range of our sample and the largest mosquitoes were the least likely to be infected with $W$. bancrofti microfilariae.

An overall increase of $0.9 \%$ in mortality rate was observed in mosquitoes fed on infected blood compared to controls up to 3-4 days post feeding. However, no statistically significant difference was found at any parasitaemia levels $\left(\chi^{2}=1.68, \mathrm{df}=1, \mathrm{P}=0.23\right)$.

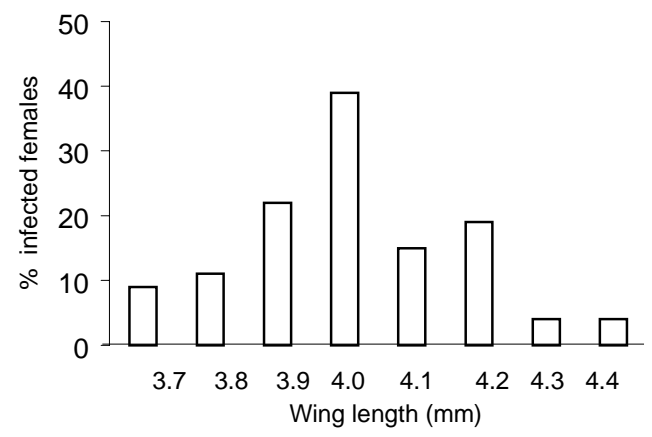

Fig. 3: frequency distribution of wing lengths of adult females of Culex quinquefasciatus infected with Wuchereria bancrofti in classes with intervals of $0.1 \mathrm{~mm}$.

\section{DISCUSSION}

Parasitic infections are generally costly to their host and this cost is often manifested as a reduction in reproductive fitness. This is so for most nematode/insect associations that have been examined (Hurd 1990). However the cost of filarial infection have been shown to be complex. A positive relationship between parasite density and degree of reproductive repression have been 
described in A. aegypti infected with Brugia pahangi (Javadian \& Macdonald 1974) and A. trivittatus infected with D. imittis (Christensen 1981) during the second gonotrophic cycle post infection. In contrast, reduction on reproductive fitness was obtained during the first gonotrophic cycle in Simulium ornatum infected with only one Onchocerca lienalis worm (Renshaw \& Hurd 1994) and no significant dose effect was observed.

Most previous studies were performed with laboratory strains of vector and may not reflect interactions that occur between a natural vector and the strain of pathogen that it transmits in the field. We thus used adults originated from field collected last stage larvae (Scorza 1972) as the source of our experimental mosquitoes and infected blood collected from subjects living in the same local. Many factors affect the number of eggs produced by mosquitoes including larval rearing conditions, adult size and age, the quality and quantity of blood ingested (Hurd et al. 1995). Although the larvae used in this study were collected from the same field site, we observed a broad range in the body size of adults that emerged and considerable variation both within and between experiments. Evidence from other studies demonstrated that, in the field, mosquito size is affected by changes in temperature, larval density, food quality and availability, which vary seasonally (Landry et al. 1988).

Overall we demonstrated a positive correlation between mosquito size and egg production in uninfected females, confirming that body size is one of the factors influencing egg production in this species, as suggested by Scorza (1972) in C. pipiens fatigans Wied. (=quinquefasciatus) from Venezuela. Highly significant positive correlation between egg production and mosquito blood meal size have been described many times in the literature (e.g. Clements \& Boocock 1984, Briegel 1990, Hurd et al. 1995). As with previous studies of vector reproduction (Hogg \& Hurd 1997) we found that this relationship was disrupted in $C$. quinquefasciatus infected with $W$. bancrofti microfilaria. However, no relationship between parasite density could be demonstrated and fertility (egg hatch) was not affected. This latter result is in agreement with Hammad (1997) who found no effect of W. bancrofti on C. quinquefasciatus (from Egypt) fertility. Unfertilized females were not investigated in this work and the absence of sperm in the spermateca must be considered.

In this study, our experimental protocol dictated that we used haematin excretion to assess the size of blood meal even though it is not as reliable a measure as haemoglobinometry (Hurd personal observations). Haematin production was highly variable and although we were not able to demonstrate a statistically significant correlation between blood meal size and egg production, our data suggests the infection may have altered this relationship. Blood sources used for feeding infected mosquitoes in this study were from various subjects. Donors may have differed with respect to age and state of health and some may have been anaemic. These parameters were not monitored but, as with differences in mosquito size, are likely to have contributed to variability between our infected groups.
The presence of malaria parasites in a bloodmeal was not found to affect the size of meal taken by anopheline mosquitoes (Hogg \& Hurd 1997, Jahan \& Hurd 1997, Ahmed et al. 1999). We have now shown that, independent of the parasitaemia levels, the bloodmeal size, taken by $C$. quinquefasciatus was not significantly affected by the presence of $W$. bancrofti. This suggests that the presence of $\mathrm{mf}$ in a bloodmeal does not inhibit feeding behaviour and that they pass through the mosquito feeding apparatus without interrupting feeding.

The variation in the effect of infection upon mosquito fecundity observed among our individual trials is difficult to explain. Haemoglobin (measured by haematin excretion) provides the major source of protein for egg production and intake of this component did not differ between infected and uninfected groups within a trial and thus is unlikely to have given rise to differences in egg production. Similarly, mosquito size did not differ between groups and is thus unlikely to have contributed to these differences. We may however be observing differences in mosquito response to a diversity of parasite population within the locality in which blood samples were collected. Alternatively, mosquito populations may vary over time with respect to their response to filarial worm infection.

Ferdig et al. (1993) took a mechanistic approach to the examination of Brugia malayi infections in Armigeres subalbatus, which is partially refractory to infection. They suggested that costs imposed by mounting an immune response, including melanization of worms, were reflected in the low tyrosine and protein levels in the ovary, the abnormal development of follicles and the retardation in oviposition time. Our previous study of this symbiosis demonstrated that there was a positive correlation between $\mathrm{mf}$ density in the donor blood and number of $\mathrm{mf}$ ingested by the female. However, the number of parasite that achieved the infective stage (L3) appeared to be independent of $\mathrm{mf}$ density in the blood, at parasitaemias ranging from 550-2,600 mf/ml (Albuquerque et al. 1999). Such difference cannot be attributed to melanization since melanized mf was only occasionally seen. We can assume that the efforts of the mosquito to eliminate the parasite and to repair tissue damage must have incurred some costs but these may not have been so great as a melanization response. Reproductive costs associated with mounting an antibacterial peptide immune response have recently been demonstrated in lipopolysaccharide injected $A$. gambiae (Ahmed et al., in press).

In the field, Lyimo and Koella (1992) found that large An. gambiae supported fewer malaria sporozoite infections than did medium sized-females. They attributed their observation to enhanced mortality rates caused by heavier parasite loads in large mosquitoes. We also found that the proportion of females infected with the filarial parasite was higher among medium sized mosquitoes than large ones. However we found no significant difference in parasite burdens of dead mosquitoes that could indicate increase in mortality of large females nor could we detect a significant effect of infection on survivorship.

C. quinquefasciatus is a natural vector of $W$. bancrofti and therefore a high degree of adaptive evolution at physiological levels may have occurred in this host-parasite 
relationship. In this mosquito species the majority of eggs laid by the females are produced during the first gonotrophic cycle (Suleman \& Reisen 1979). Therefore any metabolic benefits of shifting resources away from egg production and into storage may be available when the parasite is developing. We hypothesise that parasites that cause such an alteration of nutrient partitioning in their host may have a selective advantage. Clearly further studies need to be performed with this natural parasite/ vector association to determine whether this is so.

\section{REFERENCES}

Ahmed AM, Baggott SR, Maingon M, Hurd H. Anopheles gambiae: The fitness costs of mounting an immune response. Oikos (in press).

Ahmed AM, Maingon RD, Taylor PJ, Hurd H 1999. The effects of infection with Plasmodium yoelii nigeriensis on the reproductive fitness of the mosquito Anopheles gambiae. Invertebr Reprod Devel 36: 217-222.

Albuquerque CMR, Cavalcanti VMS, Melo MAV, Verçosa P, Regis LN, Hurd H 1999. Bloodmeal microfilariae density and the uptake and establishment of Wuchereria bancrofti infections in Culex quinquefasciatus and Aedes aegypti. Mem Inst Oswaldo Cruz 94: 591-596.

Azevedo R, Dobbin JE 1952. Filariose (Wuchereria bancrofti) no grupo residencial do IAPB no bairro de Afogados (Recife). Publ Avulsas Inst Aggeu Magalhães 1: 157-162.

Briegel H 1990. Metabolic relationship between female body size, reserves, and fecundity of Aedes aegypti. J Insect Physiol 36: 165-172.

Briegel H, Lea AO, Klowden MJ 1979. Hemoglobinometry as a method for measuring blood meal size of mosquitoes (Diptera:Culicidae). Med Entomol 5: 235-238.

Clements NA, Boocock MR 1984. Ovarian development in mosquitoes: stages of growth and arrest, and follicular resorption. Physiol Entomol 9: 1-8.

Christensen BM 1981. Effect of Dirofilaria immitis on the fecundity of Aedes trivittatus. Mosq News 41: 78-81.

Colless DH, Chellapah WT 1960. Effects of body weight and size of blood-meal upon egg production in Aedes aegypti (Linnaeus) (Diptera:Culicidae). Ann Trop Med Parasitol 54: 475-482.

Consoli RAGB, Lourenço-de-Oliveira R 1994. Principais Mosquitos de Importância Sanitária no Brasil, Fiocruz, Rio de Janeiro, $228 \mathrm{pp}$.

Ferdig MT, Beerntsen BT, Spray FJ, Li J, Christensen B 1993. Reproductive costs associated with resistance in a mosquito-filarial worm system. Am J Trop Med Hyg 49: 756762.

Forattini OP 1996. Culicidologia Médica. I Princípios Gerais Morfologia, Glossário Taxonômico, Universidade de São Paulo, São Paulo, 548 pp.

Hammad REI 1997. Effect of Wuchereria bancrofti Cobbold infection on the biological activities of Culex pipiens $\mathrm{L}$. (Diptera: Culicidae) in Egypt. J Egypt Soc Parasitol 27: 863-870.

Hogg JC, Hurd H 1995a. Plasmodium yoelii nigeriensis: the effect of high and low intensity of infection upon the egg production and bloodmeal size of Anopheles stephensi during three gonotrophic cycles. Parasitology 111: 555-562.
Hogg JC, Hurd H 1995b. Malaria-induced reduction of fecundity during the first gonotrophic cycle of Anopheles stephensi mosquitoes. Med Vet Entomol 9: 176-180.

Hogg JC, Hurd H 1997. The effects of natural Plasmodium falciparum infection on the fecundity and mortality of Anopheles gambiae s.l. in north east Tanzania. Parasitology 114: 325-331.

Hurd H 1990. Physiological and behavioural interactions between parasites and invertebrate hosts. Adv Parasitol 29: 271-318.

Hurd H, Webb TJ 1997. The role of endocrinologically active substances in mediating changes in insect hosts and insect vectors. In NE Beckage, Parasites: Effects on Host Hormones and Behaviour, Chapman and Hall, London, p. 179201.

Hurd H, Hogg JC, Renshaw M 1995. Interactions between bloodfeeding, fecundity and infection in mosquitoes. Parasitol Today 11: 411-416.

Jahan N, Hurd H 1997. The effects of infection with Plasmodium yoelii nigeriensis on the reproductive fitness of Anopheles stephensi. Ann Trop Med Parasitol 91: 365-369.

Javadian E, Macdonald WW 1974. The effect of infection with Brugia pahangi and Dirofilaria repens on the egg production of Aedes aegypti. Ann Trop Med Parasitol 68: 477-481.

Landry SV, Defoliart GR, Hogg DB 1988. Adult body size and survivorship in a field population of Aedes triseriatus. J Am Mosq Control Assoc 4: 121-128.

Lea AO, Briegel H, Lea HM 1978. Arrest, resorption or maturation of oocytes in Aedes aegypti: dependence upon the quality of the blood meal and the interval between blood meals. Physiol Entomol 3: 309-316.

Lyimo EO, Koella JC 1992. Relationship between body size of adult Anopheles gambiae s.l. and infection with the malaria parasite Plasmodium falciparum. Parasitology 104: 233237.

Maciel A, Rocha A, Marzochi KB, Medeiros Z, Carvalho AB, Regis L, Souza W, Lapa T, Furtado A 1996. Epidemiological study of bancroftian filariasis in Recife, Northeastern Brazil. Mem Inst Oswaldo Cruz 91: 449-455.

Ottesen EA, Ramachandran CP 1995. Lymphatic filariasis infection and disease: control strategies. Parasitol Today 11: 129-131.

Renshaw M, Hurd H 1994. The effect of Onchocerca infection on the reproductive physiology of the British blackfly, Simulium ornatum. Parasitology 109: 337-345.

Scorza JV 1972. Observaciones Bionómicas sobre Culex pipiens fatigans Wied., 1829 de Venezuela, Universidad de los Andes, Mérida, 198 pp.

Service MW 1996. Medical Entomology for Students, Chapman and Hall, London, 278 pp.

Sheldon BS, Verhulst S 1996. Ecological immunology: costly parasite defences and trade-offs in evolutionary ecology. Trends Ecol Evol 11: 317-321.

Suleman M, Reisen WK 1979. Culex quinquefasciatus Say: life table characteristics of adults reared from wild-caught pupae from North West Frontier province, Pakistan. Mosq News 39: 756-762.

WHO-World Health Organization 1987. Control of Lymphatic Filariasis: A Manual for Health Personnel, WHO, Geneva, $89 \mathrm{pp}$. 